\title{
Higgs constraints from vector boson fusion and scattering
}

\author{
John M. Campbell and R. Keith Ellis \\ Fermilab, \\ PO Box 500, Batavia, IL 60510, U.S.A. \\ E-mail: johnmc@fnal.gov, ellis@fnal.gov
}

\begin{abstract}
We present results on 4-lepton +2 -jet production, the partonic processes most commonly described as vector boson pair production in the Vector Boson Fusion (VBF) mode. This final state contains diagrams that are mediated by Higgs boson exchange. We focus particularly on the high-mass behaviour of the Higgs boson mediated diagrams, which unlike on-shell production, gives information about the Higgs couplings without assumptions on the Higgs boson total width. We assess the sensitivity of the high-mass region to Higgs coupling strengths, considering all vector boson pair channels, $W^{-} W^{+}$, $W^{ \pm} W^{ \pm}, W^{ \pm} Z$ and $Z Z$. Because of the small background, the most promising mode is $W^{+} W^{+}$which has sensitivity to Higgs couplings because of Higgs boson exchange in the $t$ channel. Using the Caola-Melnikov (CM) method, the off-shell couplings can be interpreted as bounds on the Higgs boson total width. We estimate the bound that can be obtained with current data, as well as the bounds that could be obtained at $\sqrt{s}=13 \mathrm{TeV}$ in the VBF channel for data samples of 100 and $300 \mathrm{fb}^{-1}$. The CM method has already been successfully applied in the gluon fusion (GGF) production channel. The VBF production channel gives important complementary information, because both production and decay of the Higgs boson occur already at tree graph level.
\end{abstract}

Keywords: Hadronic Colliders, Monte Carlo Simulations

ArXiv EPRINT: 1502.02990 


\section{Contents}

1 Introduction $\quad 1$

2 Basic rates for VBF processes $\quad 4$

3 Sensitivity to couplings and the Higgs boson width 8

$\begin{array}{lll}3.1 & \text { Limit from Run } 1 & 10\end{array}$

3.2 Limits from Runs 2 and $3 \quad 11$

$\begin{array}{llr}4 & \text { Conclusions } & 14\end{array}$

\section{Introduction}

With the advent of $13 \mathrm{TeV}$ running, the LHC will probe in greater detail the production of the Higgs boson in the sub-dominant mode - the so-called Vector Boson Fusion (VBF) mechanism. For the observed Higgs boson of mass $m_{H} \sim 125 \mathrm{GeV}$, the rate in VBF mode is calculated to be about $8 \%$ of the rate in the gluon fusion mode [1] almost independent of the energy of the LHC. Because of the increase of energy from $7 \mathrm{TeV}$ to $14 \mathrm{TeV}$ the production cross sections in the gluon fusion mode and the VBF mode are expected grow by a factor of $\sim 3.3$.

In the context of gluon fusion (GGF) production, the inadequacy of the narrow width approximation and the importance of the off-shell tail of the Higgs boson has been first noted by Kauer and Passarino [2]. They observed that more than $10 \%$ of the Higgs cross section $\sigma\left(g g \rightarrow H \rightarrow e^{-} e^{+} \mu^{-} \mu^{+}\right)$lies in the region where the four-lepton invariant mass, $m_{4 l}$ is greater than $130 \mathrm{GeV}$. The apparent breakdown of the narrow width approximation is due to the opening of the threshold for on-shell $Z$-pair production and the growth with energy of the Higgs boson amplitude.

This point was further developed by Caola and Melnikov [3] who observed that the rates for the production of the Higgs bosons were given schematically by,

$$
\begin{aligned}
& \sigma_{\text {on-shell }}\left(g g \rightarrow H \rightarrow e^{-} e^{+} \mu^{-} \mu^{+}\right) \sim \frac{g_{i}^{2} g_{f}^{2}}{\Gamma}, \\
& \sigma_{\text {off-shell }}\left(g g \rightarrow H \rightarrow e^{-} e^{+} \mu^{-} \mu^{+}\right) \sim g_{i}^{2} g_{f}^{2}
\end{aligned}
$$

where $\Gamma$ is the total width of the Higgs boson and $g_{i}, g_{f}$ are the effective couplings of the Higgs boson in the initial and final state. Thus, observation of the off-shell production gives access to information about the effective couplings, without making assumptions on the total width of the Higgs boson. Moreover, the ratio of the off-shell and on-shell rates can be used to place constraints on the total width of the Higgs boson, using accurate 
theoretical predictions for the same ratio [2-4]. This idea has been successfully used by the experiments $[5,6]$, exploiting primarily the gluon fusion production mechanism.

The Caola-Melnikov method relies on the assumption that the couplings of the Higgs bosons are the same whether they are measured on-shell or off-shell. The model independence of this assumption has been justifiably questioned in refs. [7, 8]. The importance of measuring the off-shell cross sections to constrain extensions of the standard model has been pointed out in refs. [9-12]. One of the purposes of this paper is to identify what complementary information on off-shell couplings can be obtained from VBF processes.

In the VBF processes, the high mass behaviour of the Higgs diagram has been the subject of extensive discussion because it gives access to the longitudinal modes of the vector boson. These polarizations are only present because of electroweak symmetry breaking. ${ }^{1}$ Indeed the scattering of longitudinal vector bosons was of great importance in attempts to provide an upper limit on the mass of the Higgs boson [15]. However in the Standard Model it will be extremely challenging to observe the longitudinal modes in the next run, because the vector boson polarization produced by emission from a quark is predominantly transverse $[16,17]$.

We can apply the Caola-Melnikov method to bound the Higgs boson width, using the vector boson fusion process. ${ }^{2}$ As quoted later, current constraints on the on-shell signal strength place it at about the SM value. In this case the production and decay of the Higgs boson occurs at tree level so that this process is sensitive to different theoretical systematics relative to the gluon fusion process. In particular it is not susceptible to loop effects that decouple in the off-shell region, such as those discussed in ref. [7]. Figure 1 shows a comparison of the rates for the gluon fusion process and the vector boson fusion process. As one can see from figure 1, the tail of the Higgs-mediated diagrams is relatively more important for VBF than for gluon fusion, compared to their respective peak cross sections. The differing fall off of the purely Higgs-mediated curves is due to the growth proportional to $E^{2}(E)$ of the underlying VBF (GGF) amplitudes.

The experimental study of the production of vector boson pairs by the VBF mechanism is still not fully developed. Observation of the related process $p+p \rightarrow Z+$ jet + jet has established the feasibility of the VBF method $[18,19]$. The results are found to be in agreement with Standard Model predictions [20, 21].

Evidence for the $W^{ \pm} W^{ \pm}$process at $\sqrt{s}=8 \mathrm{TeV}$ has been presented by both the ATLAS [22] and CMS [23] collaborations. The accumulated luminosity is still too low to permit stringent VBF cuts on these data samples. The $W^{ \pm} W^{ \pm}$process will play an important role in this paper because of the low backgrounds. The $W^{-} W^{+}$channel is the VBF process with the largest rate, although it has substantial backgrounds from $t \bar{t}+$ jet production. Note that although the $W^{ \pm} Z$ and $W^{ \pm} W^{ \pm}$channels do not contain diagrams with an $s$-channel Higgs boson, they are sensitive to the Higgs coupling through $t$-channel exchange diagrams. In this paper we will use the acronym VBF to denote both vector boson fusion ( $s$-channel exchange) and vector boson scattering ( $t$-channel exchange). The

\footnotetext{
${ }^{1}$ For a recent reviews with extensive references we refer the reader to refs. [13, 14].

${ }^{2}$ The suggestion that the Caola-Melnikov method can also be applied in the VBF channels $Z Z$ and $W^{+} W^{-}$has previously been made in ref. [7].
} 


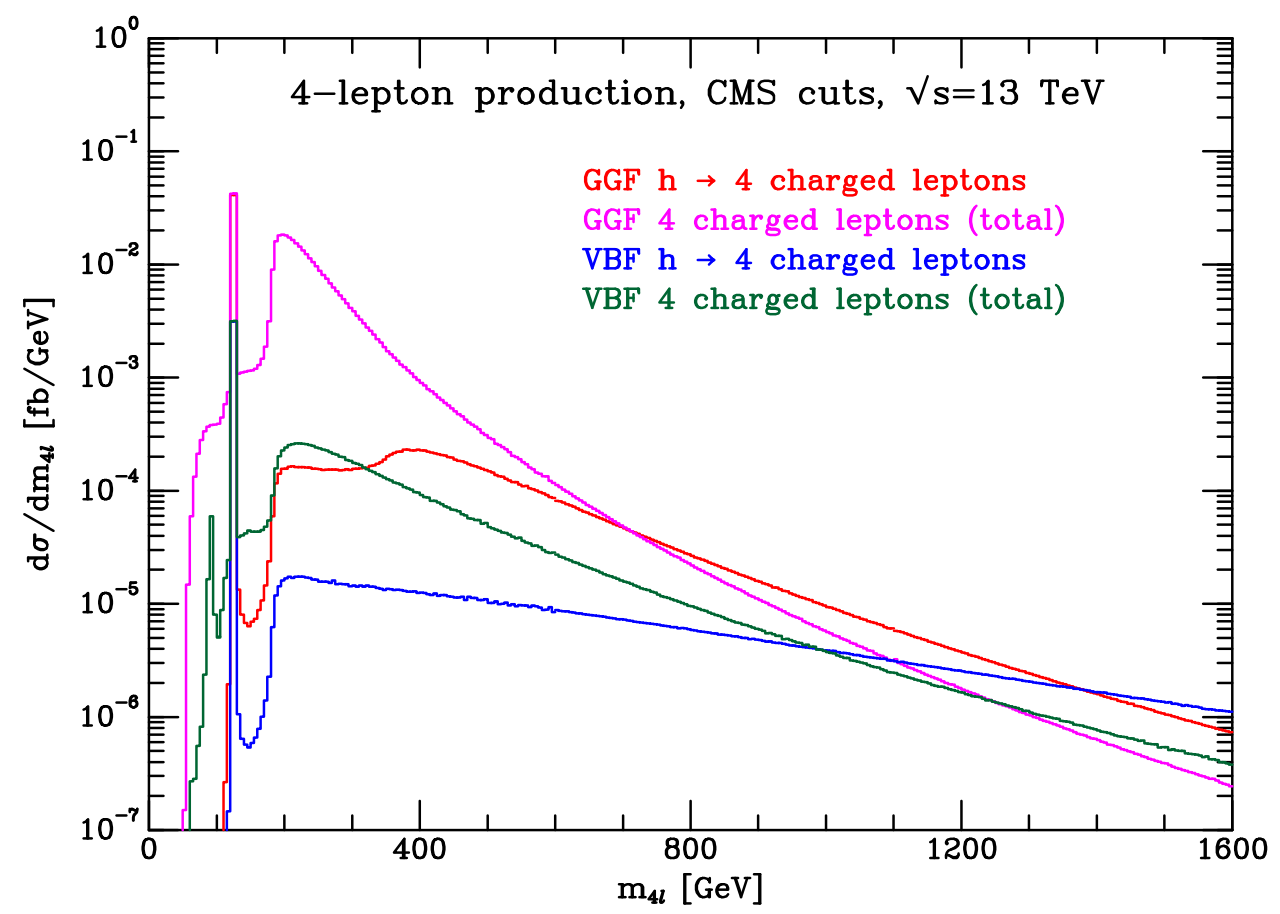

Figure 1. Comparison of the rates for the gluon fusion process $g g \rightarrow H \rightarrow e^{-} e^{+} \mu^{-} \mu^{+}$and the vector boson fusion process $q q \rightarrow e^{-} e^{+} \mu^{-} \mu^{+} q q$. The GGF curves cross at $m_{4 l}=700 \mathrm{GeV}$, whereas the VBF curves cross at $m_{4 l}=1000 \mathrm{GeV}$. The CMS cuts correspond to those used in ref. [4] and, for the VBF process, we also apply the cuts specified in eqs. (2.2), (2.5) and (2.6). The rates are plotted as a function of the four-lepton mass.

potential to study all vector boson pair channels in the next two runs will be discussed in section 2 .

In the above paragraph we have referred to the VBF processes as $W^{-} W^{+}, W^{ \pm} W^{ \pm}$, $W^{ \pm} Z$ and $Z Z$. We emphasize that this is only a shorthand for all processes that lead to the same 4-lepton final state, i.e. all doubly-, singly- and non-resonant contributions are included. The doubly resonant processes that define the explicit final states are listed below.

$$
\begin{aligned}
& q+q \rightarrow \begin{array}{l}
W^{+}+W^{-}+q+q \\
\quad \rightarrow \mu^{-}+\nu_{\mu} \\
\rightarrow \nu_{e}+e^{+}
\end{array}
\end{aligned}
$$

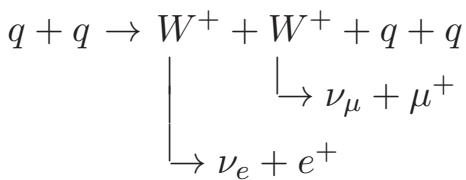

$$
\begin{aligned}
& q+q \rightarrow W^{+}+Z / \gamma+q+q \\
& \rightarrow \mu^{-}+\mu^{+} \\
& \rightarrow \nu_{e}+e^{+} \\
& q+q \rightarrow \begin{array}{c}
W^{-}+W^{-}+q+q \\
\mid \rightarrow \mu^{-}+\bar{\nu}_{\mu} \\
\rightarrow e^{-}+\bar{\nu}_{e}
\end{array}
\end{aligned}
$$




\begin{tabular}{lll} 
Run 2: & $2015-2017$ & $\sim 100 \mathrm{fb}^{-1}$ \\
Run 3: & $2019-2021$ & $\sim 300 \mathrm{fb}^{-1}$ \\
\hline
\end{tabular}

Table 1. Assumed schedule and luminosity of the LHC for the next 7 years.

$$
\begin{aligned}
& q+q \rightarrow Z / \gamma+Z / \gamma+q+q \\
& \underset{\rightarrow e^{-}+e^{+}}{\longrightarrow} \mu^{-}+\mu^{+}
\end{aligned}
$$

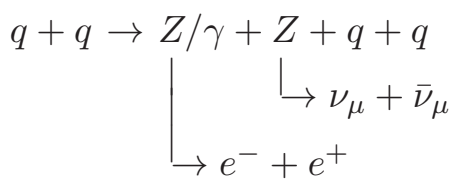

In addition to the specific leptonic final states shown, our phenomenological analysis will include other states with different flavours of leptons $(e, \mu)$ and $\left(\nu_{e}, \nu_{\mu}, \nu_{\tau}\right)$. Interferences between states with identical leptons in the final state are small and will be ignored.

\section{Basic rates for $\mathrm{VBF}$ processes}

We first want to establish which of the VBF modes will be accessible in Run 2 and Run 3 of the LHC. The size of the expected data samples is shown in table 1. For this exploratory study we will use tree-graph calculations for which representative diagrams are shown in figure 2 .

The VBF diagrams in which we are primarily interested are shown in $(a)$ and $(b)$ of the figure and contribute to the amplitude at $O\left(\alpha^{3}\right)$. There are also mixed QCD-electroweak diagrams that lead to the same final state that occur at $O\left(\alpha^{2} \alpha_{s}\right)$, shown in diagrams $(c)$ and $(d)$. These amplitudes have all been calculated and included in the parton-level integrator, MCFM [24]. These tree level amplitudes have previously been calculated in the program PHANTOM $[25,26]$ and are also available in MadGraph [27].

In principle we should consider the interference of the electroweak and QCDelectroweak amplitudes. However the leading color interference between quark-quark diagrams $(a, b)$ and $(c)$ vanishes. The interference, which is color-suppressed, can only occur between a limited number of processes with identical final state quarks. In the phase space region relevant for VBF production, it is very small [28, 29]. Note that even channels such as $W^{ \pm} W^{ \pm}$and $W^{ \pm} Z$, that do not include a $s$-channel Higgs boson contribution, are still sensitive to the Higgs boson couplings through $t$-channel exchange. The tree-level approximation should be sufficient for a preliminary idea, because the NLO corrections have been shown to be quite small for these processes [30-32].

Our results have been obtained using the parameters shown in table 2, working in the complex mass scheme [33] where $\sin ^{2} \theta_{W}$ is related to the complex masses by,

$$
\sin ^{2} \theta_{W}=1-\left(\frac{m_{W}^{2}-i m_{W} \Gamma_{W}}{m_{Z}^{2}-i m_{Z} \Gamma_{Z}}\right)
$$

This is important to preserve gauge invariance. We use the LO pdf set from MSTW2008 [34] and evaluate all cross-sections using an event-by-event scale, $\mu$, that is given by the partonic center of mass energy, $\mu=\sqrt{\hat{s}}$. 

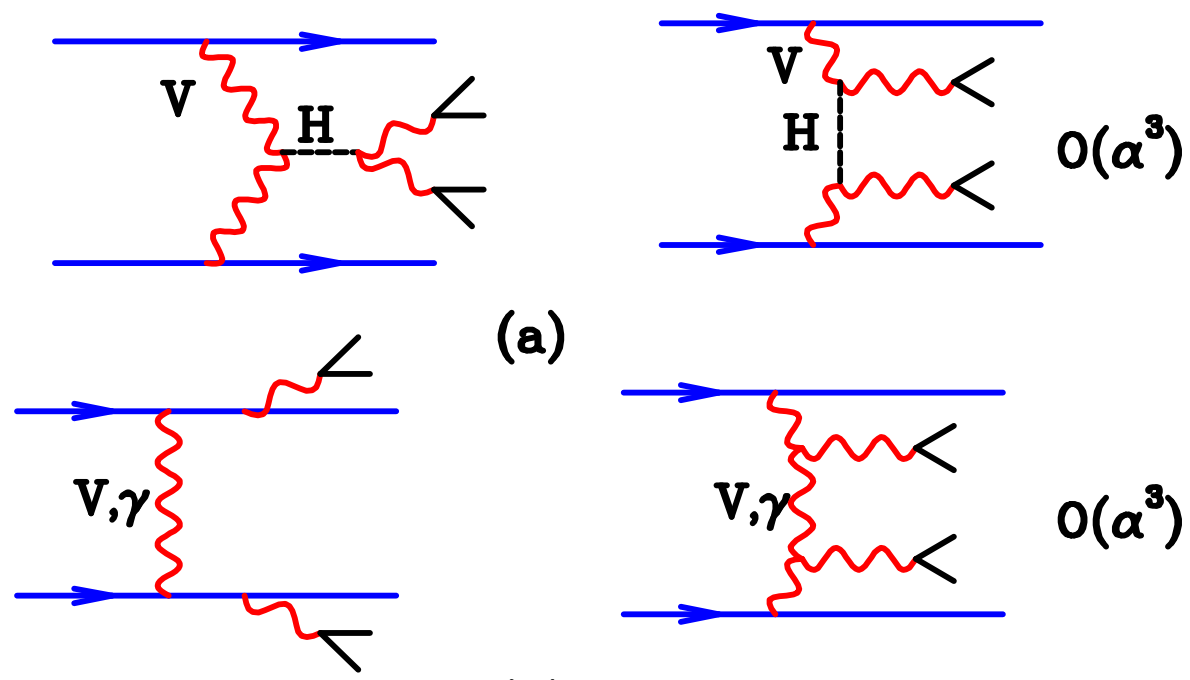

(a)

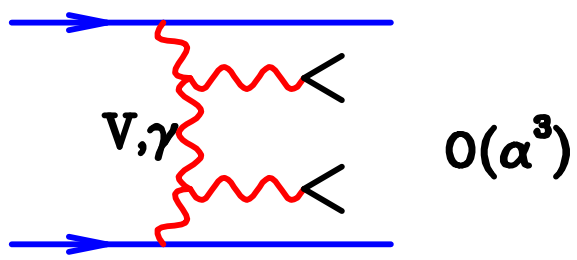

(b)

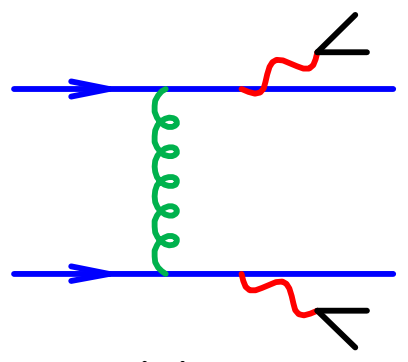

(c)

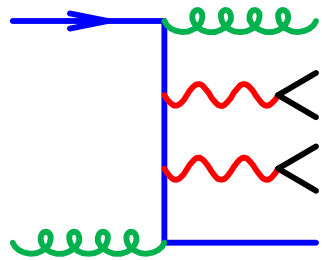

(d)

Figure 2. Representative Feynman diagrams, where $V$ denotes a $W$ or $Z$ boson.

\begin{tabular}{|c|c|c|c|}
\hline$m_{H}$ & $125 \mathrm{GeV}$ & $\Gamma_{H}$ & $0.004165 \mathrm{GeV}$ \\
$m_{W}$ & $80.419 \mathrm{GeV}$ & $\Gamma_{W}$ & $2.06 \mathrm{GeV}$ \\
$m_{Z}$ & $91.188 \mathrm{GeV}$ & $\Gamma_{Z}$ & $2.49 \mathrm{GeV}$ \\
$m_{t}$ & $173.0 \mathrm{GeV}$ & $m_{b}$ & $4.75 \mathrm{GeV}$ \\
$e^{2}$ & 0.0948355 & $g_{W}^{2}$ & 0.4267132 \\
$\sin ^{2} \theta_{W}$ & $0.22228-0.00131 i$ & $G_{F}$ & $0.116639 \times 10^{-4}$ \\
\hline
\end{tabular}

Table 2. Masses, width and Electroweak parameters used to produce the results in this paper.

In order to select topologies that enhance the vector boson scattering contributions in comparison with continuum or background contributions, specialized cuts are required. For simplicity, we first apply a set of cuts that is almost the same for all processes. The two jets are identified by the anti- $k_{T}$ algorithm,

$$
p_{T, J}>20 \mathrm{GeV},\left|\eta_{J}\right|<4.5, R=0.4 .
$$

For the leptons we apply the following cuts:

$$
\begin{aligned}
p_{T, \ell} & >20 \mathrm{GeV}, \quad\left|\eta_{\ell}\right|<2.5, \\
m_{l l} & >10 \mathrm{GeV}, \quad \text { for all charged lepton combinations. }
\end{aligned}
$$


For processes containing neutrinos the missing transverse energy satisfies,

$$
E_{T}>40 \mathrm{GeV} \text {. }
$$

The specialized cuts that isolate the VBF contributions are as follows. We ensure that the tagging jets lie in opposite hemispheres, have a rapidity gap of at least 2.5 units between them, and that the invariant mass of the jets, $m_{j_{1} j_{2}}$, is large:

$$
y_{\text {gap }}>2.5, \quad \eta_{1} \times \eta_{2}<0, \quad m_{j_{1} j_{2}}>500 \mathrm{GeV} .
$$

As a final cut, we also require that the rapidities of any charged leptons lie between the two jet rapidities,

$$
\eta_{J}^{\min }<\eta_{\ell}<\eta_{J}^{\max }
$$

We now detail the process-dependent additional cut that has been applied in each case to isolate the off-shell region. For the four charged-lepton process we cut on $m_{4 l}$, the invariant mass of the four lepton system,

$$
m_{4 l}^{2}=\left(p_{l_{1}}+p_{l_{2}}+p_{l_{3}}+p_{l_{4}}\right)^{2},
$$

while for the other $Z Z$ process we use the transverse mass $m_{T}^{Z Z}$ defined by,

$$
\left(m_{T}^{Z Z}\right)^{2}=\left[\sqrt{m_{Z}^{2}+p_{T, l l}^{2}}+\sqrt{m_{Z}^{2}+E_{T, \mathrm{miss}}^{2}}\right]^{2}-\left|\vec{p}_{T, l l}+\vec{E}_{T, \mathrm{miss}}\right|^{2} .
$$

For the $W W$ processes we define the transverse mass as,

$$
\left(m_{T}^{W W}\right)^{2}=\left[E_{T, l l}+E_{T, \text { miss }}\right]^{2}-\left|\vec{p}_{T, l l}+\vec{E}_{T, \text { miss }}\right|^{2},
$$

where $E_{T, l l}=\sqrt{p_{T, l l}^{2}+m_{l l}^{2}}$. The appropriate transverse variable for the $W Z$ processes is,

$$
\left(m_{T}^{W Z}\right)^{2}=\left[\sqrt{m_{3 l}^{2}+p_{T, 3 l}^{2}}+E_{T, \text { miss }}\right]^{2}-\left|\vec{p}_{T, 3 l}+\vec{E}_{T, \mathrm{miss}}\right|^{2} .
$$

For the two-lepton processes, $W^{-} W^{+}, W^{ \pm} W^{ \pm}$and $Z Z \rightarrow 2 l 2 \nu$, we impose additional cuts in order to reduce the backgrounds from top quark processes. We require that two combinations of lepton and jet invariant masses are above the top mass [14],

$$
m_{l_{1} j_{2}}, m_{l_{2} j_{1}}>200 \mathrm{GeV} .
$$

For these cuts $l_{1}, l_{2}\left(j_{1}, j_{2}\right)$ represent the leptons (jets) of highest and lowest transverse momentum respectively.

The processes that we will study in this paper, together with the cross sections after imposing the above set of cuts, are shown in tables 3 (electroweak process) and 4 (mixed QCD-electroweak). We also show the expected number of events in $100 \mathrm{fb}^{-1}$ of data, after all flavors of lepton have been included. Note that, in this paper, we have consistently neglected $\tau$-leptons. In principle, including $\tau$-leptons would increase the anticipated event rates, by a factor of $9 / 4$ for channels such as $W^{-} W^{+}$, but in practice the increase would 


\begin{tabular}{|l|l|l|c|c|c|}
\hline Process & $\begin{array}{l}\text { Nominal } \\
\text { process }\end{array}$ & \multicolumn{1}{c|}{ Cut } & $\begin{array}{c}\sigma[\mathrm{fb}] \\
O\left(\alpha^{6}\right)\end{array}$ & Factor & $\begin{array}{c}\text { Events } \\
\text { in } 100 \mathrm{fb}^{-1}\end{array}$ \\
\hline$p p \rightarrow e^{-} \mu^{+} \nu_{\mu} \bar{\nu}_{e} j j$ & $W^{-} W^{+}$ & $m_{T}^{W W}>300 \mathrm{GeV}$ & 0.1899 & $\mathrm{x} 4$ & 76 \\
\hline$p p \rightarrow \nu_{e} e^{+} \nu_{\mu} \mu^{+} j j$ & $W^{+} W^{+}$ & $m_{T}^{W W}>300 \mathrm{GeV}$ & 0.1358 & $\mathrm{x} 2$ & 27 \\
$p p \rightarrow e^{-} \overline{\nu_{e}} \mu^{-} \bar{\nu}_{\mu} j j$ & $W^{-} W^{-}$ & $m_{T}^{W W}>300 \mathrm{GeV}$ & 0.0440 & $\mathrm{x} 2$ & 9 \\
\hline$p p \rightarrow \nu_{e} e^{+} \mu^{-} \mu^{+} \mu^{+} j j$ & $W^{+} Z$ & $m_{T}^{W Z}>300 \mathrm{GeV}$ & 0.0500 & $\mathrm{x} 4$ & 20 \\
$p p \rightarrow e^{-} \bar{\nu}_{e} \mu^{-} \mu^{+} j j$ & $W^{-} Z$ & $m_{T}^{W Z}>300 \mathrm{GeV}$ & 0.0243 & $\mathrm{x} 4$ & 10 \\
\hline$p p \rightarrow l^{-} l^{+} \nu_{l} \bar{\nu}_{l} j j$ & $Z Z$ & $m_{T}^{Z Z}>300 \mathrm{GeV}$ & 0.0225 & $\mathrm{x} 6$ & 14 \\
$p p \rightarrow l^{-} l^{+} \nu_{l} \bar{\nu}_{l} j j$ & $Z Z$ & $m_{T}^{W W}>300 \mathrm{GeV}$ & 0.0181 & $\mathrm{x} 6$ & 11 \\
$p p \rightarrow e^{-} e^{+} \mu^{-} \mu^{+} j j$ & $Z Z$ & $m_{4 l}>300 \mathrm{GeV}$ & 0.0218 & $\mathrm{x} 2$ & 4 \\
\hline
\end{tabular}

Table 3. Electroweak $\left(\mathcal{O}\left(\alpha^{6}\right)\right)$ cross sections at $\sqrt{s}=13 \mathrm{TeV}$, under the cuts given in eqs. (2.2)(2.6) and the off-shell definition specified in the table. The factor gives the approximate number by which the result shown for specific lepton flavours must be multiplied to account for two flavours of charged leptons, $e, \mu$ and three flavours of neutral leptons, $\nu_{e}, \nu_{\mu}, \nu_{\tau}$.

not be so large because of the limited efficiency for $\tau$-identification. From the results in these tables it is clear that the final states of highest interest in the immediate future are the two charged-lepton ones. Despite being relatively background-free, the $Z Z \rightarrow 4 l$ rate is so small that the SM expectation is for only a few events. Similarly the $Z Z \rightarrow 2 l 2 \nu$ channel with a $m_{T}^{Z Z}$ cut anticipating $Z$-pair events produces only 11 events and suffers from much larger backgrounds. The $W^{-} W^{+} \rightarrow 2 l 2 \nu$ channel has a very high rate and in reality cannot be separated from the $Z Z \rightarrow 2 l 2 \nu$ channel. For this reason we consider both processes with a $m_{T}^{W W}$ cut; the event rates will be added in subsequent sections. The same-sign dilepton processes $W^{ \pm} W^{ \pm} \rightarrow 2 l 2 \nu$ are, in total, only about a factor of three smaller than the opposite-sign one. The mixed QCD-electroweak processes in table 4 represent significant, irreducible backgrounds which in most cases are of similar size as the electroweak processes. These backgrounds are reduced to this level by the imposition of the VBF cuts. The notable exceptions are once again same-sign $W^{+} W^{+}$and $W^{-} W^{-}$ production, where the pure electroweak processes are larger by an order of magnitude.

The same-sign lepton channels are relatively background-free ${ }^{3}$ and the $Z Z \rightarrow 4 l$ channel suffers only small backgrounds. However the processes involving neutrinos are potentially subject to large backgrounds from top production, possibly in association with additional jets. Further sources of background include $W+$ jets events and QCD multijets, where jets are misidentified as leptons. We do not include a study of the effects of such misidentification in this paper. To assess the importance of top quark processes we have computed the LO cross-section for $t \bar{t}$ production under the same set of cuts. We find, in the off-shell tail defined by $m_{T}^{W W}>300 \mathrm{GeV}$,

$$
\sigma\left(t \bar{t} \rightarrow e^{-} \bar{\nu}_{e} b \mu^{+} \nu_{\mu} \bar{b}\right)=0.637 \mathrm{fb}
$$

\footnotetext{
${ }^{3}$ The double parton scattering contribution is negligible with our cuts [35].
} 


\begin{tabular}{|l|l|l|c|c|c|}
\hline Process & $\begin{array}{l}\text { Nominal } \\
\text { process }\end{array}$ & Cut & $\begin{array}{c}\sigma[\mathrm{fb}] \\
O\left(\alpha^{4} \alpha_{s}^{2}\right)\end{array}$ & Factor & $\begin{array}{c}\text { Events } \\
\text { in } 100 \mathrm{fb}^{-1}\end{array}$ \\
\hline$p p \rightarrow e^{-} \mu^{+} \nu_{\mu} \bar{\nu}_{e} j j$ & $W^{-} W^{+}$ & $m_{T}^{W W}>300 \mathrm{GeV}$ & 0.2236 & $\mathrm{x} 4$ & 89 \\
\hline$p p \rightarrow \nu_{e} e^{+} \nu_{\mu} \mu^{+} j j$ & $W^{+} W^{+}$ & $m_{T}^{W W}>300 \mathrm{GeV}$ & 0.0079 & $\mathrm{x} 2$ & 2 \\
$p p \rightarrow e^{-} \overline{\nu_{e}} \mu^{-} \overline{\nu_{\mu}} j j$ & $W^{-} W^{-}$ & $m_{T}^{W W}>300 \mathrm{GeV}$ & 0.0025 & $\mathrm{x} 2$ & 0 \\
\hline$p p \rightarrow \nu_{e} e^{+} \mu^{-} \mu^{+} \mu^{+} j j$ & $W^{+} Z$ & $m_{T}^{W Z}>300 \mathrm{GeV}$ & 0.0916 & $\mathrm{x} 4$ & 37 \\
$p p \rightarrow e^{-} \overline{\nu_{e}} \mu^{-} \mu^{+} j j$ & $W^{-} Z$ & $m_{T}^{W Z}>300 \mathrm{GeV}$ & 0.0454 & $\mathrm{x} 4$ & 18 \\
\hline$p p \rightarrow l^{-} l^{+} \nu_{l} \bar{\nu}_{l} j j$ & $Z Z$ & $m_{T}^{Z Z}>300 \mathrm{GeV}$ & 0.0143 & $\mathrm{x} 6$ & 9 \\
$p p \rightarrow l^{-} l^{+} \nu_{l} \bar{\nu}_{l} j j$ & $Z Z$ & $m_{T}^{W W}>300 \mathrm{GeV}$ & 0.0118 & $\mathrm{x} 6$ & 7 \\
$p p \rightarrow e^{-} e^{+} \mu^{-} \mu^{+} j j$ & $Z Z$ & $m_{4 l}>300 \mathrm{GeV}$ & 0.0147 & $\mathrm{x} 2$ & 3 \\
\hline
\end{tabular}

Table 4. Mixed QCD-electroweak $\left(\mathcal{O}\left(\alpha^{4} \alpha_{s}^{2}\right)\right)$ cross sections at $\sqrt{s}=13 \mathrm{TeV}$, under the cuts given in eqs. (2.2)-(2.6) and the off-shell definition specified in the table.

which corresponds to 254 total events, summing over lepton flavors, in $100 \mathrm{fb}^{-1}$ of data. Although this estimate ignores both the effect of higher orders, and the application of a $b$-jet veto to suppress some of these events, it is sufficient to illustrate the severity of this background. This background rate is larger than the expected signal for $W^{-} W^{+}$production given in table 3 . Even if the background can be somewhat reduced by application of a $b$-jet veto or other cuts, it will still present a significant background with attendant uncertainties. For the same-sign channels this background is relatively insignificant since it would only enter if the charge of one of the leptons were misidentified, which typically occurs at the sub-percent level [14].

Given the size of the event rates in table 3 and the background levels discussed above, it is clear that the most important process for Run 2 is like-sign production, $W^{ \pm} W^{ \pm}$. However in Run 3, some of the other processes will also contribute useful information. In the following section we will present results for all processes for the sake of comparison. In addition, with sufficient understanding of top quark backgrounds, it may eventually be possible to extricate the opposite-sign $W^{-} W^{+}$channel from the top background. At present we are considering analyses based on cuts only. With sufficient data one can move to more sophisticated analyses, e.g. ones based on multivariate methods, that take more detailed information about the matrix elements into account.

\section{Sensitivity to couplings and the Higgs boson width}

We now turn to the matter of the sensitivity of the VBF cross sections to the Higgs boson couplings. We will work in the interim framework for the analysis of Higgs couplings [36] where the couplings of the Higgs to $W$ and $Z$ bosons scale in the same way,

$$
\frac{\Gamma_{W W}}{\Gamma_{W W}^{\mathrm{SM}}}=\kappa_{V}^{2}, \quad \frac{\Gamma_{Z Z}}{\Gamma_{Z Z}^{\mathrm{SM}}}=\kappa_{V}^{2} .
$$


As indicated before, the advantage of the off-shell measurements is that they give information about the Higgs couplings, without assumptions on the total width of the Higgs boson. Although the parameter range $\kappa_{V}<1$ is perhaps better motivated from a theoretical point of view, we shall consider both $\kappa_{V}<1$ and $\kappa_{V}>1$. The latter range comes into play when we try and constrain the total Higgs width using the CM method [3]. Within the standard model, and after the Higgs boson discovery, the Higgs couplings are completely determined. The interim framework attempts to capture BSM features in an approximate way.

As more data is accumulated, the emphasis will move from this interim framework of effective couplings, to a more general approach using higher dimension operators in an effective theory. The effective field theory operators parameterize the observed behaviour at current energies in terms of towers of higher dimension operators, normally chosen to be $\mathrm{CP}$-invariant and invariant under $\mathrm{SU}(2) \times \mathrm{U}(1)$ local symmetry. The effective operators are the consequence of unknown physics occurring at a higher scale $\Lambda$. By assumption the scale $\Lambda$ is higher than the scale currently being probed and the importance of the operators is controlled by their dimension.

A complete operator analysis is beyond the scope of this paper. We shall consider one particular operator of dimension six in order to make a connection with our interim framework,

$$
\mathcal{L}_{\mathrm{HD}}=F_{\mathrm{HD}} \operatorname{tr}\left[\mathbf{H}^{\dagger} \mathbf{H}-\frac{v^{2}}{4}\right] \cdot \operatorname{tr}\left[\left(\mathbf{D}_{\mu} \mathbf{H}\right)^{\dagger}\left(\mathbf{D}^{\mu} \mathbf{H}\right)\right] .
$$

where $\mathbf{D}=\partial_{\mu}-i g \mathbf{W}_{\mu}-i g^{\prime} \mathbf{B}_{\mu}$ is the $\mathrm{SU}(2) \otimes \mathrm{U}(1)$ covariant derivative. $\mathcal{L}_{\mathrm{HD}}$ leads to the following Feynman rules for the couplings of a single Higgs boson in the unitary gauge,

$$
\begin{aligned}
h W_{\mu}^{+} W_{\nu}^{-}: & i g M_{W} g_{\mu \nu} \frac{v^{2} F_{\mathrm{HD}}}{2}, \\
h Z_{\mu} Z_{\nu}: & i g \frac{M_{W}}{\cos ^{2} \theta_{W}} g_{\mu \nu} \frac{v^{2} F_{\mathrm{HD}}}{2} .
\end{aligned}
$$

Operators of dimension six that contribute to triple gauge boson couplings (TGC) can be ignored in the present context, since their coefficients are exquisitely constrained by TGC measurements [37]. The operator in eq. (3.2), if we ignore unitarization effects, just corresponds to an effective coupling.

$$
\kappa_{V}=1+F_{\mathrm{HD}} \frac{v^{2}}{2}
$$

where $v=0.246 \mathrm{TeV}$ and $F_{\mathrm{HD}}$ can have either sign. Note that useful reference points are obtained by choosing $F_{\mathrm{HD}}= \pm 30 \mathrm{TeV}^{-2}$, which correspond to $\kappa_{V}=2$ and $\kappa_{V}=0$ respectively. However the validity of the effective field theory is seriously compromised for a value of $F_{\mathrm{HD}}$ that large, since the corresponding scale $\Lambda$ is too low. Conversely, to probe scales higher than $1 \mathrm{TeV}$ will nominally require sensitivity to $\kappa_{V}$ at the $3 \%$ level.

The rescaling in eq. (3.1) implies that the off-shell cross section in the VBF final states should be different from the Standard Model. Due to the interference between diagrams that involve the Higgs boson and those that do not, the off-shell cross sections do not simply grow with $\kappa_{V}^{4}$, as suggested by eq. (1.1). Instead there is a term proportional to 
$\kappa_{V}^{2}$ resulting from the interference, whose coefficient is negative because the interference is destructive. Explicitly, we find the number of off-shell events in $100 \mathrm{fb}^{-1}$, due to the electroweak process only, is given by,

$$
\begin{aligned}
l^{-} l^{+} \nu \bar{\nu}: & N^{\mathrm{off}}=108.9-42.6 \kappa_{V}^{2}+20.8 \kappa_{V}^{4} \\
l^{+} l^{+} \nu \nu: & N^{\mathrm{off}}=37.2-18.3 \kappa_{V}^{2}+8.3 \kappa_{V}^{4} \\
l^{-} l^{-} \bar{\nu} \bar{\nu}: & N^{\mathrm{off}}=11.0-4.1 \kappa_{V}^{2}+1.8 \kappa_{V}^{4} \\
l^{+} l^{-} l^{+} \nu: & N^{\mathrm{off}}=23.5-6.7 \kappa_{V}^{2}+3.2 \kappa_{V}^{4} \\
l^{+} l^{-} l^{-} \bar{\nu}: & N^{\mathrm{off}}=11.5-3.3 \kappa_{V}^{2}+1.6 \kappa_{V}^{4} \\
l^{-} l^{+} l^{-} l^{+}: & N^{\mathrm{off}}=6.0-3.0 \kappa_{V}^{2}+1.5 \kappa_{V}^{4}
\end{aligned}
$$

where, for the $l^{-} l^{+} \nu \bar{\nu}$ channel we have added the $W^{-} W^{+}$and $Z Z$ results for $m_{T}^{W W}>$ $300 \mathrm{GeV}$ from earlier, with the appropriate multiplicative factors. Adding in the irreducible background from the mixed QCD-electroweak processes in table 4 we obtain,

$$
\begin{aligned}
l^{-} l^{+} \nu \bar{\nu}: & N^{\text {off }}=205.8-42.6 \kappa_{V}^{2}+20.8 \kappa_{V}^{4} \\
l^{+} l^{+} \nu \nu: & N^{\text {off }}=38.8-18.3 \kappa_{V}^{2}+8.3 \kappa_{V}^{4} \\
l^{-} l^{-} \bar{\nu} \bar{\nu}: & N^{\text {off }}=11.5-4.1 \kappa_{V}^{2}+1.8 \kappa_{V}^{4} \\
l^{+} l^{-} l^{+} \nu: & N^{\text {off }}=60.0-6.7 \kappa_{V}^{2}+3.2 \kappa_{V}^{4} \\
l^{+} l^{-} l^{-} \bar{\nu}: & N^{\text {off }}=29.6-3.3 \kappa_{V}^{2}+1.6 \kappa_{V}^{4} \\
l^{-} l^{+} l^{-} l^{+}: & N^{\text {off }}=8.9-3.0 \kappa_{V}^{2}+1.5 \kappa_{V}^{4}
\end{aligned}
$$

Note that the numbers of events are not so different for $\kappa_{V}=0$, (no Higgs boson) and for $\kappa_{V}=1$, (SM). This reflects the fact that, for this energy and luminosity, we cannot place the off-shell mass cut at a value sufficiently far above the electroweak scale, $v=246 \mathrm{GeV}$ that the terms that fail to cancel for $\kappa_{V} \neq 1$ dominate.

\subsection{Limit from Run 1}

The existing analyses of $W^{ \pm} W^{ \pm}$production in $\sqrt{s}=8 \mathrm{TeV}$ running by both the ATLAS [22] and CMS [23] collaborations already provide a constraint on the Higgs boson couplings and width. Since the luminosity accumulated in Run 1 is too low to clearly isolate the VBF region, the expected sensitivity is rather limited.

As an example, we have repeated our analysis using the cuts presented in ref. [22]. We find that the cross-section for two electrons (or positrons), computed in the fiducial region defined there, is:

$$
\sigma_{\text {electron }}^{\text {same-sign }}=0.345-0.036 \kappa_{V}^{2}+0.014 \kappa_{V}^{4} \mathrm{fb} .
$$

This does not yet account for multiple lepton flavors or efficiencies. We therefore normalize to the SM expectation quoted by ATLAS, $\sigma=0.95 \pm 0.06 \mathrm{fb}$, to obtain,

$$
\sigma_{\text {fiducial }}^{\text {same-sign }}=1.015-0.106 \kappa_{V}^{2}+0.040 \kappa_{V}^{4} \mathrm{fb} .
$$

The fiducial-region measurement quoted by ATLAS is,

$$
\sigma^{\text {measured }}=1.3 \pm 0.4(\text { stat }) \pm 0.2(\text { syst }) \mathrm{fb} .
$$


Accounting for a $2 \sigma$ deviation in the sum of statistical, systematic and theoretical uncertainties we thus obtain the coupling constraint,

$$
\kappa_{V}<7.8
$$

We now turn to the matter of the sensitivity of the VBF cross sections to the Higgs boson width, under the assumption that all Higgs boson couplings and widths scale in a manner that leaves the on-shell cross sections unchanged. This assumption is supported by the latest analyses of Run 1 LHC data from ATLAS and CMS that constrain the on-shell signal strength $\mu=\sigma^{\text {observed }} / \sigma^{\mathrm{SM}}$. By analysing the $H \rightarrow W W$ decay channel, the ATLAS experiment obtains the following bound [38],

$$
\mu_{\mathrm{VBF}}^{\mathrm{ATLAS}}=1.27_{-0.45}^{+0.53}
$$

while the CMS experiment finds [39],

$$
\mu_{\mathrm{VBF}}^{\mathrm{CMS}}=1.16_{-0.34}^{+0.37}
$$

In order for the on-shell signal strength $\mu$ to be equal to unity, this implies that the width of the Higgs boson should scale as,

$$
\Gamma_{H} \rightarrow \kappa_{V}^{4} \Gamma_{H}^{\mathrm{SM}}
$$

Making the simplifying assumption that the signal strength is exactly equal to one with no error, we obtain an upper-bound on the Higgs boson width of,

$$
\Gamma_{H}<60.8 \times \Gamma_{H}^{\mathrm{SM}} .
$$

This simple analysis has many shortcomings; it is a leading order analysis without matching experimental cuts for the on-shell $W^{-} W^{+}$and the off-shell $W^{ \pm} W^{ \pm}$samples. We present it only to make the basic point that off-shell couplings may be best constrained by the $W^{ \pm} W^{ \pm}$ channels, despite the fact they do not contain the $s$-channel exchange of a Higgs boson.

\subsection{Limits from Runs 2 and 3}

To assess the utility of the VBF processes in the immediate run, we first perform a simple analysis to determine the optimal cut to isolate the off-shell tail. In this analysis we obtain the SM prediction for the number of events $(N)$ for all values of the off-shell $m_{\text {cut }}$, where the cut variable depends on the process under consideration. For the 4-charged lepton process we require $m_{4 l}>m_{\text {cut }}$, eq. (2.7), and for the other processes we use eqs. (2.8), (2.9), (2.10) as appropriate. The SM expectation includes both the electroweak and the mixed QCDelectroweak processes, although not the (small effects of) interference between these two types of process. At $\sqrt{s}=8 \mathrm{TeV}$ there is significant additional background in the $W^{ \pm} W^{ \pm}$ channel, categorized in ref. [22], as prompt, conversions and non-prompt backgrounds. We have no way of estimating the importance of these backgrounds at $\sqrt{s}=13 \mathrm{TeV}$, in particular since some of them are detector dependent. In the following we shall assume that they can be subtracted with zero error. Our analysis of the width also ignores any errors 


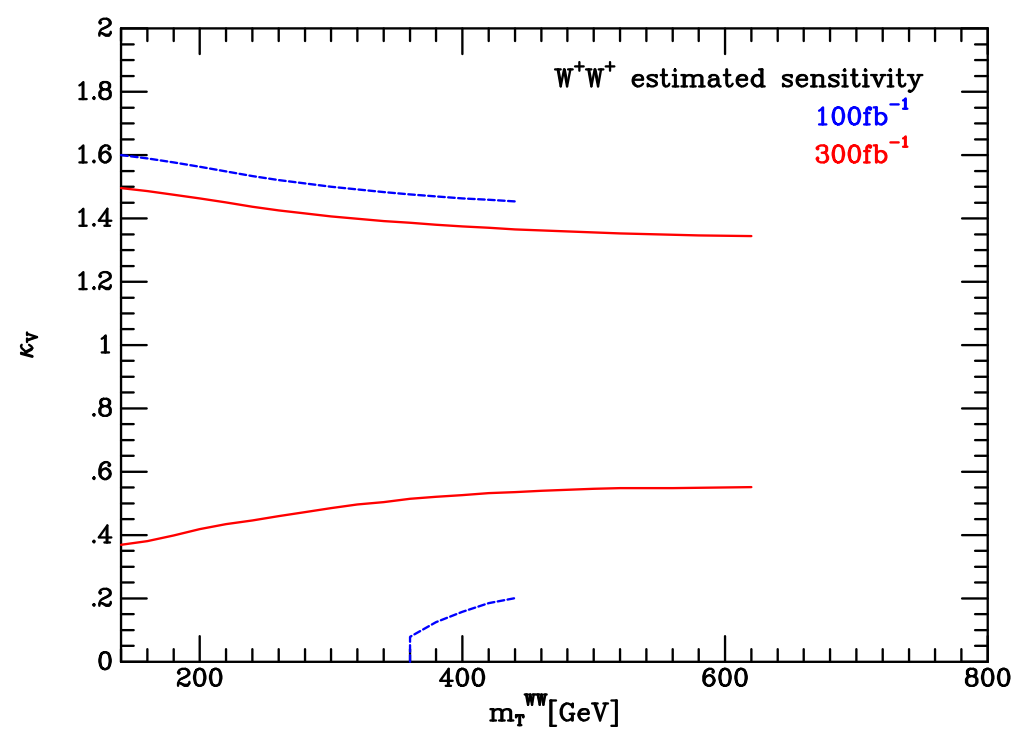

Figure 3. The upper and lower bounds on $\kappa_{V}$ obtained from $W^{+} W^{+}$events, as a function of the cut on the transverse mass, $m_{T}^{W W}$. The bounds are obtained as described in the text. Limits from $100 \mathrm{fb}^{-1}$ of data are shown as dashed (blue) lines and those from $300 \mathrm{fb}^{-1}$ are indicated by solid (red) lines.

in the on-shell cross section measurement due to the more serious backgrounds present in this case.

Defining a purely statistical uncertainty by $\delta_{N}=\sqrt{N}$, we consider which values of $\kappa_{V}$ could be excluded at approximately $95 \%$ c.l. by an observation of $N+2 \delta_{N}$ events. Since the lower bound on $\kappa_{V}$ is most useful, we then choose the value of $m_{\text {cut }}$ that provides the strongest such bound, $\kappa_{V}>\kappa_{V}^{\min }$. If no lower bound is obtained we optimize with respect to the upper bound. In all cases, we ensure that the value of $m_{\text {cut }}$ corresponds to a SM prediction of at least 10 events. This process is repeated for samples of both 100 and $300 \mathrm{fb}^{-1}$ of data.

With this simple procedure we find that, with $100 \mathrm{fb}^{-1}$ of data, only the $W^{+} W^{+}$ process provides a lower bound. The limits are shown in figure 3. This data set is statistically limited and we find that the best limit corresponds to the largest cut allowed before the SM expectation falls below 10 events. At this value of the cut, $m_{\text {cut }}=440 \mathrm{GeV}$, we find,

$$
0.20<\kappa_{V}<1.45
$$

The upper bounds obtained from the other processes are,

$$
\kappa_{V}<(1.44,1.72,1.68,1.82,1.75)
$$

for, respectively, the processes $\left(W^{-} W^{+}, W^{-} W^{-}, W^{+} Z, W^{-} Z, Z Z\right)$. Figure 3 also shows the corresponding bounds expected with $300 \mathrm{fb}^{-1}$ of data, where a similar pattern is observed. The best lower limit comes again from the $W^{+} W^{+}$process and corresponds to saturating the 10 -event minimum, which is now reached with $m_{\text {cut }}=620 \mathrm{GeV}$, and 


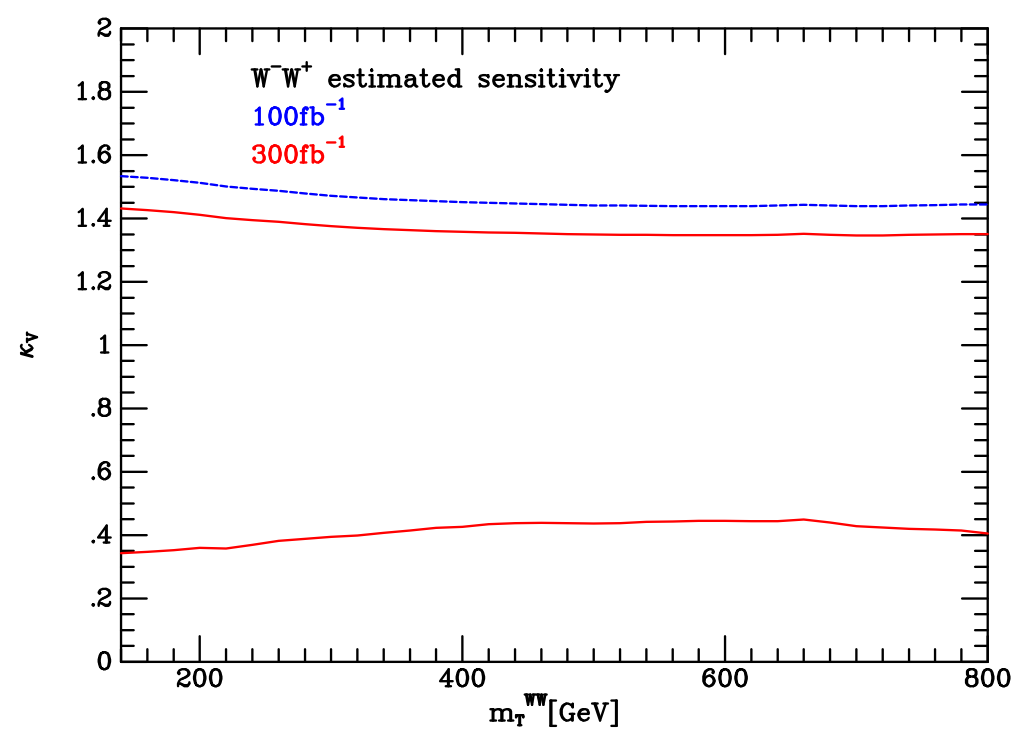

Figure 4. The upper and lower bounds on $\kappa_{V}$ obtained from $W^{-} W^{+}$events, as a function of the cut on the transverse mass, $m_{T}^{W W}$. Limits from $100 \mathrm{fb}^{-1}$ of data are shown as dashed (blue) lines and those from $100 \mathrm{fb}^{-1}$ are indicated by solid (red) lines.

we find,

$$
0.55<\kappa_{V}<1.34
$$

With a bigger data set $\left(300 \mathrm{fb}^{-1}\right)$ we can also obtain lower bounds from the $W^{-} W^{+}$ process. The sensitivity is indicated in figure 4 and we find,

$$
0.45<\kappa_{V}<1.35, \quad W^{-} W^{+}\left(m_{T}^{W W}>660 \mathrm{GeV}\right)
$$

The optimum for the $W^{-} W^{+}$channel displays a real trade-off as $m_{\text {cut }}$ is increased, between decreasing statistics and increasing sensitivity. Upper limits on $\kappa_{V}$ from the other processes are weaker.

We now turn to the matter of the sensitivity of the VBF cross sections to the Higgs boson width, under the same assumptions as discussed previously, cf. section 3.1. The best upper limits on $\kappa_{V}$ are obtained from the $W^{+} W^{+}$process. Converting these into an expected bound on the width from this channel we obtain,

$$
\begin{array}{ll}
\Gamma_{H}<4.4 \times \Gamma_{H}^{\mathrm{SM}} & \left(100 \mathrm{fb}^{-1} \text { data }\right) \\
\Gamma_{H}<3.2 \times \Gamma_{H}^{\mathrm{SM}} & \left(300 \mathrm{fb}^{-1} \text { data }\right)
\end{array}
$$

Figure 5 shows the dependence of the limit on the number of excess events for $100 \mathrm{fb}^{-1}$ and $300 \mathrm{fb}^{-1}$, in the ranges $m_{T}^{W W}>440 \mathrm{GeV}$ and $m_{T}^{W W}>620 \mathrm{GeV}$ respectively. These bounds roughly correspond to the current constraints from the gluon-fusion channel obtained in Run 1 of the LHC [5, 6]. Previous estimates of the bounds possible with even higher luminosity are given in ref. [7]. 


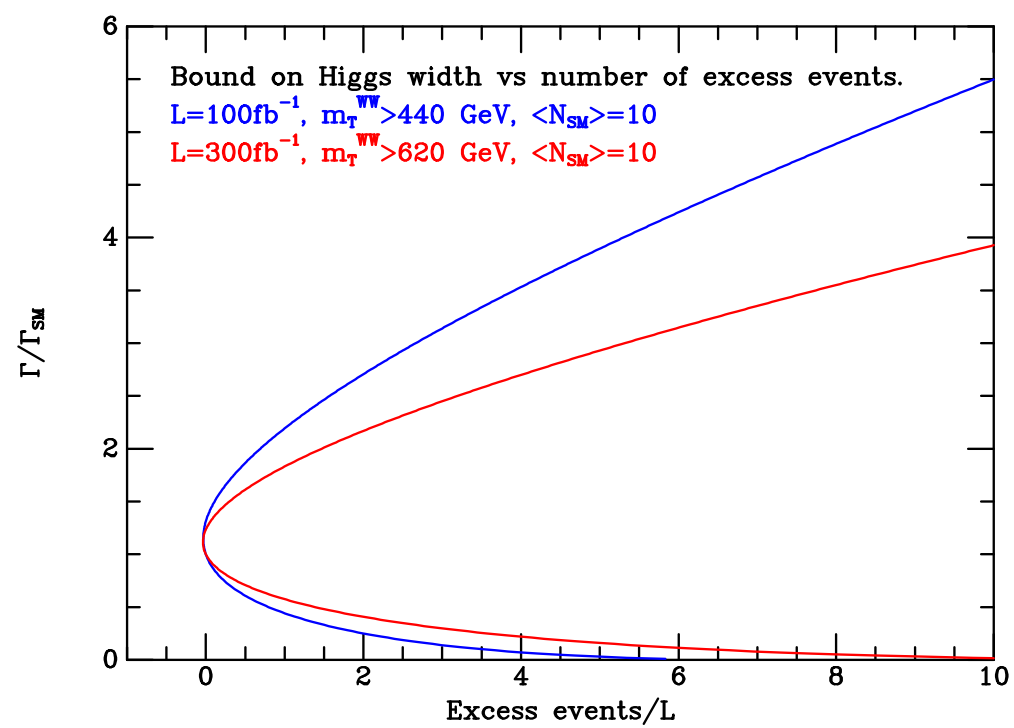

Figure 5. Bound on the Higgs width vs number of excess off-shell events in 100 and $300 \mathrm{fb}^{-1}$

\section{Conclusions}

We refocus attention on the fact that in Runs 2 and 3 at the LHC there will be sensitivity to the electroweak production of four lepton final states in the VBF mode. The most important channel will be $W^{+} W^{+}$, which despite the absence of an $s$-channel Higgs pole, still has sensitivity to the Higgs coupling.

As emphasized by Caola and Melnikov [3], the high mass tail in these final states will give access to information about the couplings of the Higgs bosons which is free from assumptions about the total width of the Higgs boson. In the first instance this information will be analysed in the effective coupling approximation which parameterizes the strength of the couplings to vector bosons as a multiple $\kappa_{V}$ of their standard model strength. Because of interference with non-Higgs mediated contributions, the sensitivity to $\kappa_{V}$ decreases as the Standard model value $\kappa_{V}=1$ is approached. We find that for the event samples likely to be accumulated in the next decade, the constraints on the effective couplings are quite modest, and consequently a full effective operator analysis of the constraints from these channels is probably premature.

Conversely, if we assume that the off-shell Higgs couplings are the same as the on-shell Higgs couplings, we can use the size of the off-shell contributions to place bounds on the total width of the Higgs boson. We have pointed out that the off-shell contributions can also be measured in channels such as $W^{ \pm} W^{ \pm}, W^{ \pm} Z$ where there is no Higgs boson peak. These have smaller backgrounds than the dominant $W^{-} W^{+}$channel. Although, as we have seen, the most stringent single channel is $W^{ \pm} W^{ \pm}$, results from all channels could be combined in the effective coupling framework.

\section{Acknowledgments}

This research is supported by the US DOE under contract DE-AC02-07CH11359. We are happy to acknowledge useful discussions with Estia Eichten and Chris Quigg. 
Open Access. This article is distributed under the terms of the Creative Commons Attribution License (CC-BY 4.0), which permits any use, distribution and reproduction in any medium, provided the original author(s) and source are credited.

\section{References}

[1] LHC Higgs Cross Section Working Group collaboration, S. Dittmaier et al., Handbook of LHC Higgs Cross Sections: 1. Inclusive Observables, arXiv:1101.0593 [INSPIRE].

[2] N. Kauer and G. Passarino, Inadequacy of zero-width approximation for a light Higgs boson signal, JHEP 08 (2012) 116 [arXiv:1206.4803] [INSPIRE].

[3] F. Caola and K. Melnikov, Constraining the Higgs boson width with ZZ production at the LHC, Phys. Rev. D 88 (2013) 054024 [arXiv:1307.4935] [INSPIRE].

[4] J.M. Campbell, R.K. Ellis and C. Williams, Bounding the Higgs width at the LHC using full analytic results for $g g \rightarrow e^{-} e^{+} \mu^{-} \mu^{+}$, JHEP 04 (2014) 060 [arXiv: 1311.3589] [INSPIRE].

[5] CMS collaboration, Constraints on the Higgs boson width from off-shell production and decay to Z-boson pairs, Phys. Lett. B 736 (2014) 64 [arXiv:1405.3455] [INSPIRE].

[6] ATLAS collaboration, Determination of the off-shell Higgs boson signal strength in the high-mass ZZ final state with the ATLAS detector, ATLAS-CONF-2014-042 (2014).

[7] C. Englert and M. Spannowsky, Limitations and Opportunities of Off-Shell Coupling Measurements, Phys. Rev. D 90 (2014) 053003 [arXiv:1405.0285] [inSPIRE].

[8] H.E. Logan, Hiding a Higgs width enhancement from off-shell gg $\left(\rightarrow h^{*}\right) \rightarrow Z Z$ measurements, arXiv:1412.7577 [INSPIRE].

[9] A. Azatov, C. Grojean, A. Paul and E. Salvioni, Taming the off-shell Higgs boson, Zh. Eksp. Teor. Fiz. 147 (2015) 410 [arXiv: 1406.6338] [InSPIRE].

[10] G. Cacciapaglia, A. Deandrea, G. Drieu La Rochelle and J.-B. Flament, Higgs couplings: disentangling New Physics with off-shell measurements, Phys. Rev. Lett. 113 (2014) 201802 [arXiv: 1406.1757] [INSPIRE].

[11] J.S. Gainer, J. Lykken, K.T. Matchev, S. Mrenna and M. Park, Beyond Geolocating: Constraining Higher Dimensional Operators in $H \rightarrow 4 \ell$ with Off-Shell Production and More, Phys. Rev. D 91 (2015) 035011 [arXiv:1403.4951] [INSPIRE].

[12] C. Englert, Y. Soreq and M. Spannowsky, Off-Shell Higgs Coupling Measurements in BSM scenarios, arXiv: 1410.5440 [INSPIRE].

[13] W. Kilian, T. Ohl, J. Reuter and M. Sekulla, High-Energy Vector Boson Scattering after the Higgs Discovery, arXiv:1408.6207 [INSPIRE].

[14] M. Szleper, The Higgs boson and the physics of $W W$ scattering before and after Higgs discovery, arXiv:1412.8367 [INSPIRE].

[15] B.W. Lee, C. Quigg and H.B. Thacker, Weak Interactions at Very High-Energies: The Role of the Higgs Boson Mass, Phys. Rev. D 16 (1977) 1519 [inSPIRE].

[16] S. Dawson, The Effective W Approximation, Nucl. Phys. B 249 (1985) 42 [INSPIRE].

[17] G.L. Kane, W.W. Repko and W.B. Rolnick, The Effective $W^{ \pm}, Z^{0}$ Approximation for High-Energy Collisions, Phys. Lett. B 148 (1984) 367 [INSPIRE]. 
[18] ATLAS collaboration, Measurement of the electroweak production of dijets in association with a Z-boson and distributions sensitive to vector boson fusion in proton-proton collisions at $\sqrt{s}=8 \mathrm{TeV}$ using the ATLAS detector, JHEP 04 (2014) 031 [arXiv: 1401.7610] [INSPIRE].

[19] CMS collaboration, Measurement of electroweak production of two jets in association with a $Z$ boson in proton-proton collisions at $\sqrt{s}=8 \mathrm{TeV}$, Eur. Phys. J. C 75 (2015) 66 [arXiv:1410.3153] [INSPIRE].

[20] B. Jager, S. Schneider and G. Zanderighi, Next-to-leading order QCD corrections to electroweak Zjj production in the POWHEG BOX, JHEP 09 (2012) 083 [arXiv:1207.2626] [INSPIRE].

[21] A. Denner, L. Hofer, A. Scharf and S. Uccirati, Electroweak corrections to lepton pair production in association with two hard jets at the LHC, JHEP 01 (2015) 094 [arXiv:1411.0916] [INSPIRE].

[22] ATLAS collaboration, Evidence for Electroweak Production of $W^{ \pm} W^{ \pm} j j$ in pp Collisions at $\sqrt{s}=8 \mathrm{TeV}$ with the ATLAS Detector, Phys. Rev. Lett. 113 (2014) 141803 [arXiv:1405.6241] [INSPIRE].

[23] CMS collaboration, Study of vector boson scattering and search for new physics in events with two same-sign leptons and two jets, Phys. Rev. Lett. 114 (2015) 051801 [arXiv: 1410.6315] [INSPIRE].

[24] J.M. Campbell and R.K. Ellis, MCFM for the Tevatron and the LHC, Nucl. Phys. Proc. Suppl. 205-206 (2010) 10 [arXiv: 1007.3492] [InSPIRE].

[25] A. Ballestrero, A. Belhouari, G. Bevilacqua, V. Kashkan and E. Maina, PHANTOM: A Monte Carlo event generator for six parton final states at high energy colliders, Comput. Phys. Commun. 180 (2009) 401 [arXiv:0801.3359] [InSPIRE].

[26] A. Ballestrero, D.B. Franzosi and E. Maina, Vector-Vector scattering at the LHC with two charged leptons and two neutrinos in the final state, JHEP 06 (2011) 013 [arXiv: 1011.1514] [INSPIRE].

[27] J. Alwall et al., The automated computation of tree-level and next-to-leading order differential cross sections and their matching to parton shower simulations, JHEP 07 (2014) 079 [arXiv: 1405.0301] [INSPIRE].

[28] J.R. Andersen and J.M. Smillie, QCD and electroweak interference in Higgs production by gauge boson fusion, Phys. Rev. D 75 (2007) 037301 [hep-ph/0611281] [INSPIRE].

[29] M. Ciccolini, A. Denner and S. Dittmaier, Electroweak and QCD corrections to Higgs production via vector-boson fusion at the LHC, Phys. Rev. D 77 (2008) 013002 [arXiv: 0710.4749] [INSPIRE].

[30] J. Baglio et al., Release Note - VBFNLO 2.7.0, arXiv:1404.3940 [InSPIRE].

[31] B. Jager and G. Zanderighi, NLO corrections to electroweak and QCD production of $W^{+} W^{+}$ plus two jets in the POWHEGBOX, JHEP 11 (2011) 055 [arXiv:1108.0864] [INSPIRE].

[32] B. Jager and G. Zanderighi, Electroweak $W^{+} W^{-} j j$ prodution at NLO in QCD matched with parton shower in the POWHEG-BOX, JHEP 04 (2013) 024 [arXiv:1301.1695] [INSPIRE].

[33] A. Denner, S. Dittmaier, M. Roth and D. Wackeroth, Predictions for all processes $e^{+} e^{-} \rightarrow 4$ fermions $+\gamma$, Nucl. Phys. B 560 (1999) 33 [hep-ph/9904472] [InSPIRE]. 
[34] A.D. Martin, W.J. Stirling, R.S. Thorne and G. Watt, Parton distributions for the LHC, Eur. Phys. J. C 63 (2009) 189 [arXiv:0901.0002] [InSPIRE].

[35] J.R. Gaunt, C.-H. Kom, A. Kulesza and W.J. Stirling, Same-sign W pair production as a probe of double parton scattering at the LHC, Eur. Phys. J. C 69 (2010) 53 [arXiv: 1003.3953] [INSPIRE].

[36] LhC Higgs Cross Section Working Group collaboration, S. Heinemeyer et al., Handbook of LHC Higgs Cross Sections: 3. Higgs Properties, arXiv:1307.1347 [INSPIRE].

[37] T. Corbett, O.J.P. Éboli, J. Gonzalez-Fraile and M.C. Gonzalez-Garcia, Determining Triple Gauge Boson Couplings from Higgs Data, Phys. Rev. Lett. 111 (2013) 011801 [arXiv: 1304.1151] [INSPIRE].

[38] ATLAS collaboration, Observation and measurement of Higgs boson decays to $W W^{*}$ with the ATLAS detector, arXiv:1412.2641 [INSPIRE].

[39] CMS collaboration, Precise determination of the mass of the Higgs boson and tests of compatibility of its couplings with the standard model predictions using proton collisions at 7 and $8 \mathrm{TeV}$, arXiv: 1412.8662 [INSPIRE]. 